\title{
Impact of PECVD SiON stoichiometry and post-annealing on the silicon surface passivation
}

\author{
J. Dupuis $^{1 *}$, E. Fourmond ${ }^{1}$, J.F. Lelièvre ${ }^{1}$, D. Ballutaud ${ }^{2}$, M. Lemiti ${ }^{1}$ \\ ${ }^{1}$ Institut des Nanotechnologies de Lyon, UMR 5270, INSA de Lyon, 7 avenue Jean Capelle, Bât. Blaise Pascal, \\ 69621 Villeurbanne Cedex, France \\ ${ }^{2}$ Groupe d'Etude de la Matière Condensée, UMR 8635, CNRS, 1 place Aristide Briand, 92195 Meudon Cedex,
} France

\section{Abstract}

Hydrogenated silicon oxynitride $(\mathrm{SiON})$ could be used in combination with silicon nitride (SiN) to create multilayer antireflection coatings for silicon solar cells. It could also be used as a passivation layer, especially on the back side of the cell.

This work deals with the passivation effect obtained on silicon surface by SiON layer deposited by Low Frequency Plasma Enhanced Chemical Vapour Deposition (LF-PECVD). SiON layers of different compositions have been deposited by varying the gas flow mixture $\left(\mathrm{NH}_{3}, \mathrm{SiH}_{4}\right.$ and $\left.\mathrm{N}_{2} \mathrm{O}\right)$ in the reactor. Infrared and X-Ray photon-electron spectroscopy were made to determine the chemical structure of SiON layer. Minority carrier lifetimes were measured by the photoconductance decay method (PCD) before and after a rapid thermal anneal. Effective lifetime, measured on 5 ohm.cm FZ-silicon wafers, can reach up to several hundreds microseconds, depending on the stoichiometry of the SiON layer. Low oxygen content samples (close to SiN layer) exhibit a good surface passivation of $250 \mu$ s but after annealing, this value is critically reduced to $6 \mu \mathrm{s}$. The opposite situation is observed for oxygen-rich layers: the effective lifetime increases from $10 \mu$ s to $150 \mu \mathrm{s}$. These behaviours could be partly explained by the composition of $\mathrm{SiON}$, the evolution of the main peaks values of the FTIR spectra and the disappearance of $\mathrm{Si}-\mathrm{H}$ bonds with anneal.

\footnotetext{
"Corresponding author. Tel.: +33-472-438-233; Fax: +33-472-738-531.

E-mail address : julien.dupuis@insa-Iyon.fr (J. Dupuis)
} 
Keywords: SiON, surface passivation, solar cells, XPS, FTIR

\section{Introduction}

Hydrogenated silicon oxynitride $\left(\mathrm{SiO}_{\mathrm{x}} \mathrm{N}_{\mathrm{y}}: \mathrm{H}\right.$ abbreviated $\mathrm{SiON}$ afterwards) can be deposited at low temperature with Plasma Enhanced Chemical Vapour Deposition (PECVD). It exhibits low absorbance and adjustable refractive index between 1.45 and 1.9 [1] by changing the ratio of precursor gas flow during deposition. SiON is thus suitable for optoelectronic devices such as waveguides, and has become a standard in optoelectronics industry [2, 3].

However, these properties can also be suitable for photovoltaic industry especially for antireflection multi-layers in combination with hydrogenated silicon nitride $\left(\mathrm{SiN}_{\mathrm{x}}: \mathrm{H}\right.$ abbreviated SiN afterwards). Adjusting carefully optical indexes and thicknesses of the film could optimise the penetration of solar photon in the solar cells. Thus double SiON/SiN layers seem to give a better transmission coefficient than a single SiN layer [4]. Gradual antireflection layers could also be imagined, with refractive index varying from $\mathrm{SiN}$ to $\mathrm{SiO}$ value to increase solar cells efficiency [5].

Antireflection layer is not the only possible application of SiON. Due to the increasing price of silicon material, standard solar cells are getting thinner and the quality of the rear surface passivation is becoming more and more crucial to maintain high solar cell efficiency [6]. The passivation effect of SiON is very promising for solar cell rear surface and could replace the actual aluminium back surface field, responsible for high constraint on thin solar cells. Moreover SiON can also be included in a stack system with $\mathrm{SiN}$ and $\mathrm{SiOx}: \mathrm{H}$ (abbreviated $\mathrm{SiO}$ afterwards) for optimum rear surface passivation [6]. Finally, low SiON indexes make this layer suitable to act as a rear surface reflector. Indeed, high wavelength photons are absorbed less efficiently by silicon and increasing their reflection at the back side of the solar cells could increase the optical path length through the silicon wafer and give them a better chance to be absorbed. 
This work aims to study electronic and structural properties of SiON deposited by PECVD for solar cells applications. The passivation impact of the layers is quantified by minority carrier lifetime (MCL) measurements, performed for different stoichiometries of SiON. The chemical structure of SiON is analysed by X-Ray photon-electron spectroscopy (XPS) and Fourier Transform Infrared spectroscopy (FTIR). All theses characterisations have been made before and after a rapid thermal anneal (RTA).

\section{Experimental}

SiON layers have been deposited with ammonia, pure silane and nitrous oxide (respectively $\mathrm{NH}_{3}, \mathrm{SiH}_{4}$ and $\mathrm{N}_{2} \mathrm{O}$ ) as precursors gases in a semi-industrial Low Frequency PECVD reactor (LF-PECVD) at $440 \mathrm{kHz}$. The reactor used has been described elsewhere [4]: it was developed by SEMCO-Engineering and presents a vertical configuration chamber. The deposition was made at $370^{\circ} \mathrm{C}$, with a power of $0.26 \mathrm{~W}$ per $\mathrm{cm}^{2}$ and a pressure of 1500 mTorr. Silane flow and total gas flow have been set respectively to $25 \mathrm{sccm}$ and $800 \mathrm{sccm}$ and maintained constant.

Different SiON stoichiometries were obtained by varying the "Ox" ratio which is defined as:

$$
O x=\frac{\mathrm{N}_{2} \mathrm{O}}{\mathrm{N}_{2} \mathrm{O}+\mathrm{NH}_{3}}
$$

The Ox ratio is related to the oxygen content of the $\mathrm{SiON}$ layer $(\mathrm{Ox}=0$ if it is pure $\mathrm{SiN}$ and $\mathrm{Ox}=1$ if it's pure $\mathrm{SiO}$ ). After deposition, refractive index, extinction coefficient and thickness were measured by spectroscopic ellipsometer (Jobin Yvon UVISEL) using Tauc Lorentz dispersion model [7].

Then XPS have been performed on SiON samples to obtain their atomic composition. Substrates were 2" CZ n-type silicon (100 oriented) with a resistivity of 1-10 Ohm.cm. The SiON layer thickness is around 70nm. The XPS measurements were carried out in a VG 220i XL system, with a base pressure of $5 \times 10^{-10}$ torr and using AlK (1486.5 eV) X-ray 
monochromatized radiation with pass energy of $20 \mathrm{eV}$ (resolution $0.2 \mathrm{eV}$ ). Energy levels of XPS were calibrated with Au single crystals. The spectra were processed using the VG Eclipse Datasystem. The background contribution is included in the fitting process, using the Shirley method.

Infrared measurements were also carried out to study structural bonds in SiON samples. For that case, substrates were FZ 2" p-type silicon with both sides polished and 4-7 ohm.cm resistivity (100 oriented). The thickness of deposited SiON films is around 200nm. The experimental setup is a Fourier-Transform infrared spectrometer (FTS60 A, Bio-Rad Inc.) associated with a DTGS detector operating in the spectrum range 1.6-20 micrometer. Next, minority carrier lifetime measurements (MCL) have been carried out to evaluate the surface passivation quality. MCL have been determined by photoconductance decay measurements using a WCT-100 Photoconductance Tool from Sinton Consulting [8]. SiON layers were deposited on both sides of polished 1-10 ohm.cm silicon wafers n-type and p-type (100 oriented) with a thickness around $70 \mathrm{~nm}$. The high quality of the silicon wafer implies that the effective measured lifetime depends essentially on the surface recombination velocity (bulk minority carrier lifetime is superior to $3 \mathrm{~ms}$ ). After all these measurements, FTIR and MCL samples were annealed at $800^{\circ} \mathrm{C}$ in a lamp furnace with a nitrogen atmosphere during several seconds, to simulate the firing of the contacts on both sides of solar cells and then new measurements were made.

\section{Ellipsometric measurements}

Refractive indexes of the different SiON layers are presented in figure 1. They vary from 1.84 for $\mathrm{SiN}$ to 1.54 for $\mathrm{SiO}$ at wavelength of $633 \mathrm{~nm}$. All other samples have their optical index between these two values. The increase of oxygen content leads to the decrease of refractive index. Moreover, there is a linear connection between the Ox ratio and the refractive index of 
the layer which allows to adjust easily the refractive index of the deposited layer by setting $\mathrm{N}_{2} \mathrm{O}$ and $\mathrm{NH}_{3}$ gases flow.

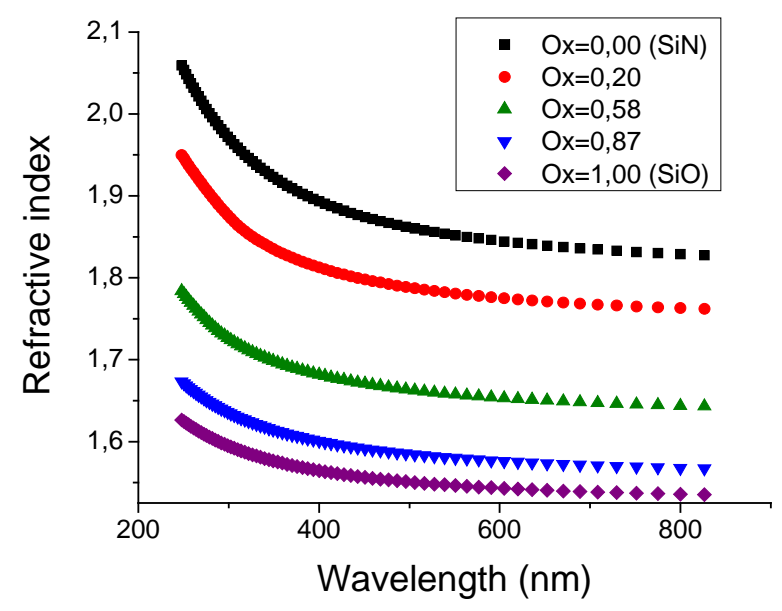

Figure 1. Wavelength-dependant refractive index of different $\mathrm{SiON}$ layers according to the gas flow ratio Ox.

The corresponding extinction coefficient $\mathrm{k}$ values are extremely low. Tauc Lorentz model gives an extinction coefficient equal to zero for wavelength superior to $300 \mathrm{~nm}$, therefore in the entire visible spectra. Null or low k is very important for front surface application in solar cells in order to minimize high-energy photons absorption in the antireflection coating itself.

\section{XPS measurements}

The complete composition of SiON samples is shown in table 1.

\begin{tabular}{|c|c|c|c|}
\hline Ox ratio & \multicolumn{3}{|c|}{ Atomic composition (20 $\mathbf{~ m}$ depth in the layer) } \\
\hline \multirow{3}{*}{0,00} & $\mathbf{O} \%$ & $\mathbf{N} \%$ & Si\% \\
\cline { 2 - 4 } 0,20 & 6,3 & 40,2 & 53,5 \\
0,58 & 23,5 & 31,4 & 45,1 \\
1,00 & 47,1 & 11,9 & 41,0 \\
& 60,8 & 1,2 & 38,0 \\
\hline
\end{tabular}

Table 1. Atomic composition of the SiON layer according to XPS measurements and analysis

As expected, the increase of $\mathrm{N}_{2} \mathrm{O}$ flow leads to high oxygen content in the SiON layer while nitrogen is decreasing in the same proportion. On the other hand silicon concentrations remain 
globally constant beyond 20 percent of oxygen in the film and suggest that nitrogen atoms bonded with silicon are replaced by oxygen atom. This behaviour has also been reported using $\mathrm{N}_{2} \mathrm{O}$ and $\mathrm{SiH}_{4}$ gas precursors $[9,10]$.

Moreover, the oxygen concentration in the layer varies linearly with the refractive index. Therefore, there is a linear connection between the Ox ratio and the atomic oxygen concentration found by XPS.

In the plasma, oxygen radicals react in priority with $\mathrm{Si}$ radicals because the most favourable chemical reaction is $\mathrm{Si}-\mathrm{O}$ bonding. $\mathrm{N}-\mathrm{H}, \mathrm{Si}-\mathrm{H}$ and $\mathrm{Si}-\mathrm{N}$ bonding seems to form when oxygen becomes deficient [9].

The hydrogen content could not be detected by XPS measurements but we can estimate its concentration around $30 \%$ for $\mathrm{SiN}$ layer and several percent for $\mathrm{SiO}$ layer $[10,11]$. For photovoltaic applications, this hydrogen is beneficial, particularly for multicrystalline silicon wafers for which a rapid thermal anneal can lead to the diffusion of atomic hydrogen and the subsequent passivation of defects in the silicon bulk [4, 12].

\section{Fourier transform infrared spectroscopy}

FTIR spectra are shown in figure 2.

The predominant absorption band is localised between $800 \mathrm{~cm}^{-1}$ and $1200 \mathrm{~cm}^{-1}$ and can be resolved into $\mathrm{Si}-\mathrm{N}$ and $\mathrm{Si}-\mathrm{O}$ groups $[9,13]$. Other absorption peaks are less significant and the $2000-4000 \mathrm{~cm}^{-1}$ spectral region was magnified five times for appreciation. Going from $\mathrm{Ox}=0$ $(\mathrm{SiN})$ to $\mathrm{Ox}=1(\mathrm{SiO})$, the main peak moves from the $\mathrm{Si}-\mathrm{N}$ stretching mode to the $\mathrm{Si}-\mathrm{O}$ stretching and rocking modes. These peaks values are shifting with the modified chemical environment from $870 \mathrm{~cm}^{-1}$ to $1030 \mathrm{~cm}^{-1}$ with the increase of oxygen content in the layer. The peaks localised at $1180 \mathrm{~cm}^{-1}$ and $3350 \mathrm{~cm}^{-1}$ correspond to $\mathrm{N}-\mathrm{H}$ bending and stretching vibration respectively [14]. These peaks disappear with the decrease of nitrogen content in the 
layer. Si-H stretching mode stands at $2200 \mathrm{~cm}^{-1}$ and is disappearing too with the increase of oxygen in the sample. Finally, the small peak at $3500 \mathrm{~cm}^{-1}$ which appears for rich oxygen layer could be related to $\mathrm{H}-\mathrm{O}-\mathrm{H}$ stretching vibration [15].

With the increase of oxygen content, the layer chemical configuration is deeply changed. The variation in peaks intensity with the Ox ratio could be related to the privileged Si-O bond formation. This is why $\mathrm{Si}-\mathrm{N}, \mathrm{N}-\mathrm{H}$ and $\mathrm{Si}-\mathrm{H}$ related peaks are all decreasing whereas $\mathrm{Si}-\mathrm{O}$ is increasing.

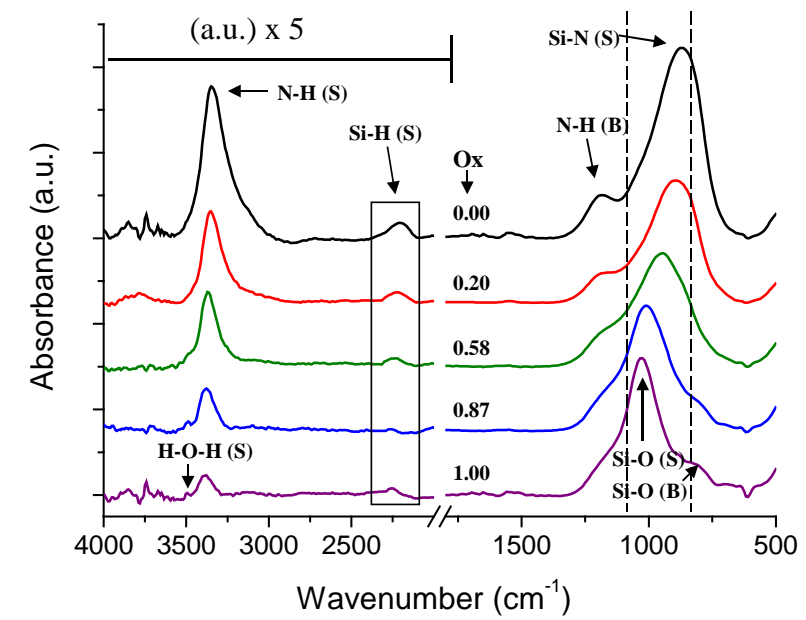

(a)

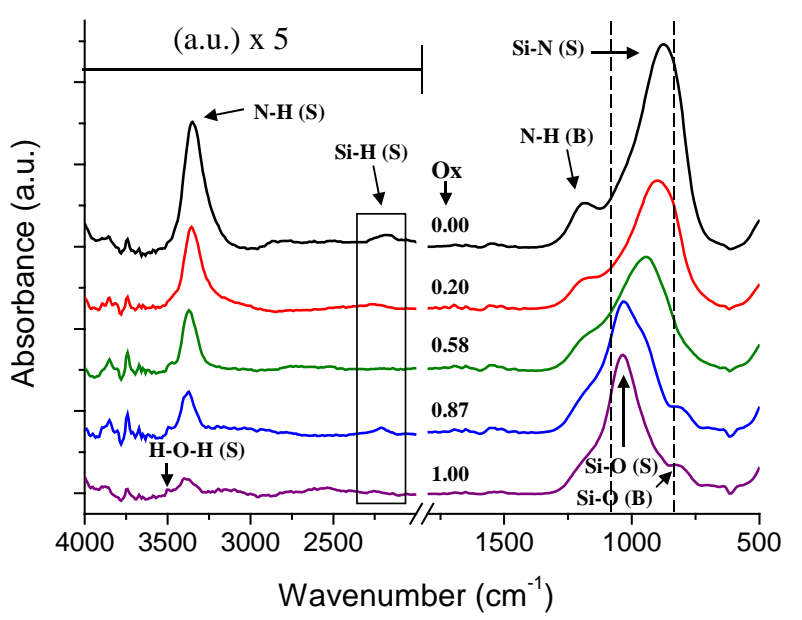

(b)

Figure 2. FTIR spectra of SiON layers before (a) and after (b) anneal as function of Ox ratio indicated above each spectrum. The dotted lines indicate the position of the Si-N stretching vibration mode in $\mathrm{Si}_{3} \mathrm{~N}_{4}\left(835 \mathrm{~cm}^{-1}\right)$ and the $\mathrm{Si}-\mathrm{O}$ stretching vibration mode in thermal $\mathrm{SiO}_{2}$ $\left(1075 \mathrm{~cm}^{-1}\right)$, respectively $[16,17]$. The framed area points out the change in Si-H stretching bond according to the Ox ratio, before and after the rapid thermal anneal step.

After the rapid thermal anneal (RTA), no change is observed on optical index or thickness. Annealing is normally followed by the densification of the SiON films [15] but the RTA seems too short and the temperature seems too low to densify SiON layers. FTIR spectra (Fig. 2b) are very similar to the previous ones. Still, Si-O stretching vibration tends to shift to a 
higher wavenumber for oxygen-rich sample. For example, looking at the Ox=1 spectrum, SiO peak shifts from $1027 \mathrm{~cm}^{-1}$ to $1040 \mathrm{~cm}^{-1}$. On the other hand, the reduction of $\mathrm{Si}-\mathrm{H}$ stretching vibration is clearly visible for nitride-rich sample.

The energy brought by RTA leads to bonding re-arrangement within the layers. Si-H bonds are sufficiently weak $(3,3 \mathrm{eV})$ to be broken easily by the RTA, contrary to N-H $(4,0 \mathrm{eV})$ or Si$\mathrm{N}(3,7 \mathrm{eV})$ which stays almost unaffected [4] [18]. Therefore, hydrogen might escape from the layer and the shifts of all Si-O stretching vibration could be explain by the silicon dangling bonds which bind to oxygen atoms in priority because in $\mathrm{SiON}, \mathrm{Si}-\mathrm{O}$ bond is more stable and more thermodynamically privileged than other bonds.

\section{Minority carrier lifetime measurements (MCL)}

The evolution of MCL according to the refractive index is represented in figure 3. For comparison, MCL of SiN obtained in the same PECVD reactor has been plot with SiON results. SiN results are discussed elsewhere [19].

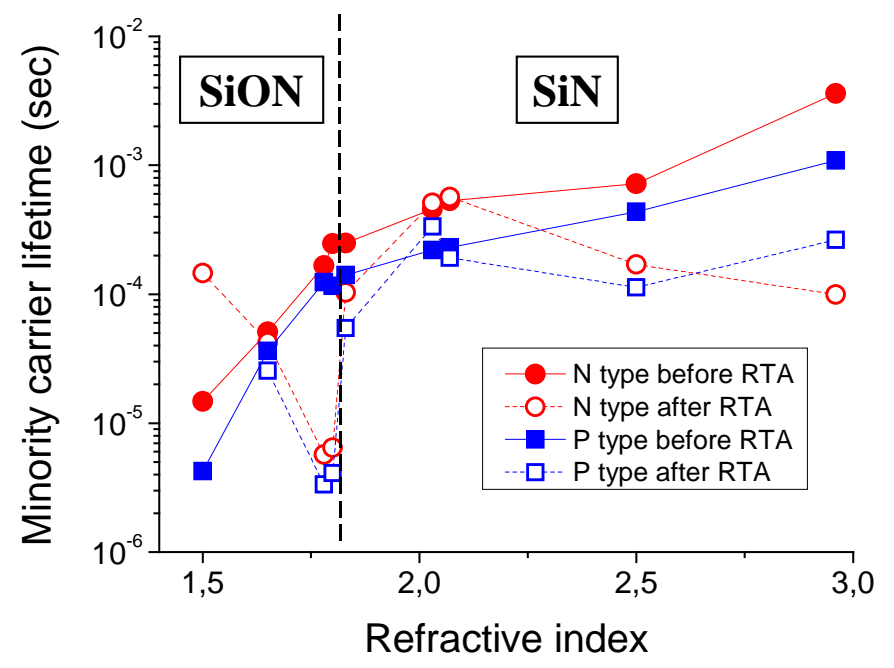

Figure 3. Evolution of minority carrier lifetime for different $\mathrm{SiN}$ and $\mathrm{SiON}$ layers according to the refractive index at $\lambda=633 \mathrm{~nm}$ and for an excess carrier concentration of $7.10^{14} \mathrm{~cm}^{-3}$. Lines are guide for the eye. 
Before RTA, MCL is decreasing with the refractive index for $\mathrm{N}$ and $\mathrm{P}$ substrates. MCL is always higher for n-type silicon. This behaviour could be explain by of the higher cross capture section of electron in p-type silicon [20].

Increasing oxygen content leads to decrease nitrogen and hydrogen content in the layers and form in priority Si-O bonds. But, contrary to thermal oxide, it seems that $\mathrm{Si}-\mathrm{O}$ bonds formed at low temperature do not create a passivation effect as good as other bonds like $\mathrm{Si}-\mathrm{N}$ or $\mathrm{Si}-\mathrm{H}$ : the value of MCL is a hundred time lower for $\mathrm{SiO}$ layer than for SiN. Therefore, it seems that the increase of oxygen is not good for the surface passivation.

The deterioration of the passivation could be explained by the fact that the field effect due to the fixed charges density in $\mathrm{SiON}$ is lower than for $\mathrm{SiN}$, in particular because of the decrease of $\mathrm{K}+$ centres [22].

Moreover, the interface state density $\mathrm{D}_{\mathrm{it}}$, which is a critical parameter for passivation, has been reported to be thirty time higher for $\mathrm{SiN}$ film than for thermal $\mathrm{SiO}_{2}$ film [21]. In $\mathrm{SiN}$ layer, a part of the passivation quality depends on the hydrogen content in the layer which could limits $\mathrm{D}_{\mathrm{it}}$ value [4]. However, hydrogen content in SiON films has been reported to decrease when oxygen increases [10] [11]. This may explain an increase of $\mathrm{D}_{\mathrm{it}}$ which could not be neutralised by the as-deposited oxide: it would result of a continuous decrease of MCL as oxygen content increases in the layer.

After RTA the situation is completely different. MCL of nitrogen-rich SiON layers become very low whereas it is the opposite in oxygen-rich $\mathrm{SiON}$ samples.

FTIR measurements have shown a slight shift for all Si-O stretching bonds towards the Si-O wavenumber value in thermal oxide. These shifts could suggest a modified environment of SiO stretching vibration and maybe a better passivation behaviour. This could explain the observed MCL improvement after RTA for oxygen-rich SiON layers. On the other hand, the 
re-arrangement of oxygen bonds seems to be insufficient to explain by itself these high MCL improvements. Moreover, for nitrogen-rich sample, the sudden decrease of MCL with RTA remains difficult to explain. A possible explanation could be that for $\mathrm{SiN}$ or nitrogen-rich $\mathrm{SiON}$, FTIR spectra shows that RTA leads to the break of $\mathrm{Si}-\mathrm{H}$ bonds and free hydrogen in the layer. This hydrogen could exodiffuse or combine with elements of the layer. However, bonds formations which occur at the interface between the film and the substrate could be beneficial for the surface passivation quality (decrease Dit). Therefore, it is possible that the decrease of silicon concentration for Ox ratio inferior to 0.20 (see table 1) leads to a higher porosity of the SiON film compared to SiN layer [11]. This would enhance the exodiffusion of hydrogen, and thus limit the passivation effect of the layer [23].

\section{Conclusion}

Silicon oxynitride (SiON) were grown by LF-PECVD technique by varying flows of $\mathrm{SiH}_{4}$, $\mathrm{NH}_{3}$ and $\mathrm{N}_{2} \mathrm{O}$ gas precursors. Refractive indexes obtained vary between 1.54 and 1.84 and absorption is inexistent in the full visible spectral range. The composition of these layers determined by XPS shows the increase of oxygen content related to the increase of $\mathrm{N}_{2} \mathrm{O}$ gas flow : oxygen radicals react mainly with silicon, prior to hydrogen or nitrogen. The refractive index has been found directly proportional to the oxygen content. FTIR spectra have confirmed this tendency and have pointed out the re-arrangement of SiON films with a rapid thermal anneal (RTA). Minority carrier lifetime (MCL) measurements have shown that oxygen and hydrogen play a major role in the passivation quality of the SiON layer. High oxygen content leads to a bad surface passivation before RTA maybe because of the poor passivation properties of deposited oxide and because the reduced amount hydrogen leads to increase $\mathrm{D}_{\mathrm{it}}$. After RTA the situation is totally opposite: oxygen-rich samples exhibit a better MCL because the Si-O properties change a little and seems to tend to the thermal oxide behaviour. One the other hand nitrogen-rich samples have a very low lifetime, which is 
difficult to interpret. This could be explained by the relatively high porosity of the SiON layer, leading to hydrogen exodiffusion.

C-V measurements, hydrogen desorption and RTA with different temperatures are in progress to understand better these behaviours.

Finally, MCL results show that oxygen-rich SiON films are very promising to be used as an alternative passivation layer of the rear surface of silicon solar cells.

\section{Acknowledgements}

The authors want to thanks J. Randrianalisoa from the CETHIL for FTIR characterization.

\section{References}

[1] A. del Prado, E. San Andrés, F.L. Martínez, I. Mártil, G. González-Dĺaz, W. Bohne, J. Röhrich, B. Selle, M. Fernández, Vacuum 67 (2002), 507-512.

[2] Y. Wang, X. Cheng, Z. Lin, C. Zhang, F. Zhang, Vacuum 72 (2004), 345-349.

[3] K. Wörhoff, A. Driessen, P.V. Lambeck, L.T.H. Hilderink, P.W.C. Linders, Th.J.A. Popma, Sensors and Acuators 74 (1999), 9-12.

[4] J.F. Lelièvre, PhD Thesis, Institut des Nanotechnologies de Lyon, INSA de Lyon, France, 2007.

[5] M. Lipiński, A. Kaminski, J.F. Lelièvre, M. Lemiti, E. Fourmond, P. Zieba, Physica Status Solidi 4 (2007), 1566-1569.

[6] M. Hofmann, E. Schneiderlöchner, W. Wolke, R. Preu, $19^{\text {th }}$ European Photovoltaic Solar Energy Conference Proceeding, Paris, France, 7-11 June, 2004, 1037-1040.

[7] G.E. Gellison, Jr and F.A. Modine, Applied Physics Letters 69 (1996), 371-373.

[8] R.Sinton, A.Cuevas Appl. Phys. Lett., 69(17), 1996, 2510.

[9] M.I. Alayo, I. Pereyra, W.L. Scopel, M.C.A. Fantini, Thin Solid Films 402 (2002) 154161. 
[10] S. Naskar, PhD Thesis, Case Western Reserve University, Cleveland, United States, 2006.

[11] M. Józwik, P. Delobelle, C. Gorecki, A. Sabac, L. Nieradko, C. Meunier, F. Munnik, Thin Solid Films 468 (2004) 84-92.

[12] A.G. Aberle, Progress in Photovoltaics : Research and Applications 8 (2000) 473-487.

[13] I.V. Afanasyev-Charkin, L.G. Jacobsohn, R.D. Averitt, M. Nastasi, Journal of Vacuum Science and Technology 22 (2004) 2342-2346.

[14] E. Bustarret, M. Bensouda, M.C. Habrard, J. C. Bruyère, Physical Review B38 (1988) $8171-8183$

[15] F. Ay, A. Aydinli, Optical Materials 26 (2004) 33-46.

[16] I. Pereyra, M.I. Alayo, Journal of Non-Crystalline Solids 212 (1997) 225-231.

[17] S. Dreer, P. Wilhartitz, Pure and Applied Chemistry 76 (2004) 1161-1213.

[18] B. deB. Darwent, National Standard Reference Data Series, National Bureau of Standards, No. 31, Washington, DC, 1970.

[19] J.F. Lelièvre, Y. Rozier, A. Bernaudeau, O. Palais, A. Kaminski, S. Quoizola, O. Nichiporuk, M. Bérenguer, P. Girard, J-C. Loretz, C. Giral, Y. Pellegrin, M. Lemiti, $15^{\text {th }}$ International Photovoltaic Science and Engineering Conference \& Solar Energy Exhibition, Shanghai, China, 10-15 October, 2005, 124-125.

[20] S. Wolf, G. Agostinelli, G. Beaucarne, Journal of Applied Physics 97 (2005) 063303.

[21] F.M Schuurmans, PhD Thesis, Utrecht university, the Netherlands, 1998.

[22] A.G. Aberle, Solar Energy Materials \& Solar Cells 65 (2001) 239-248.

[23] H.F.W. Dekkers, L. Carnel, G. Beaucarne, W. Beyer, 20th European Photovoltaic Solar Energy Conference, Barcelona, Spain, 6-10 June, 2005, 721-724. 